Recepción: 24 / 10 / 2016

Aceptación: 02 / 01 / 2017

Publicación: 18 / 05 / 2017

Ciencias de la educación

Artículo de investigación

\title{
La terapia del lenguaje, fonoaudiología o logopedia
}

\section{The therapy of language, fonoaudiology or logopedia}

\author{
Terapia da fala, a terapia da fala ou terapia da fala
}

\author{
Martha A. Ayala-Paredes ${ }^{\mathrm{I}}$ \\ aliciadevillagrán@ hotmail.com \\ Nelly Z. Pluas-Arias ${ }^{\mathrm{I}}$ \\ zolypa@hotmail.com \\ Segundo F. Pacherres-Seminario ${ }^{\text {III }}$ \\ segundo.pacherress@ug.edu.ec
}

Correspondencia: aliciadevillagrán@ hotmail.com

\begin{abstract}
' Magister en Gerencia Educativa, Especialista en Gestión de Procesos Educativos, Diplomado en Docencia Superior, Psicóloga Industrial, Docente de la Universidad Estatal de Guayaquil, Guayaquil, Ecuador.

${ }^{1}$ Magister en Diseño Curricular, Profesora de Segunda Enseñanza Especialización: Informática, Licenciada en Ciencias de la Educación Especialización: Informática, Licenciada en Laboratorio Clínico, Docente de la Universidad Estatal de Guayaquil, Guayaquil, Ecuador.

${ }^{\text {II }}$ Magister en Docencia Universitaria e Investigación Educativa, Psicólogo Clínico, Diplomado en Docencia Superior, Docente de la Universidad Estatal de Guayaquil, Guayaquil, Ecuador.
\end{abstract}





\section{Resumen}

La apertura de espacios para la persona con discapacidad es tangible si se gesta desde el interior de las organizaciones formales e informales. Exige un trabajo permanente en la estructuración de diseños intergrupales, credibilidad institucional y una intencionalidad de las comunidades que comparten la problemática del individuo y que aúnan esfuerzos para una cualificación de su vida en un plano de equidad respecto a sus congéneres. A tales efectos se realiza el presente estudio con la finalidad de abordar la temática de la terapia del lenguaje, en el mismo se abordan aspectos relacionados con el lenguaje, sus características, trastornos. Definición, objetivos de la terapia del lenguaje, habilidades terapéuticas y algunos resultados de investigaciones realizadas.

Palabras clave: lenguaje; terapia; terapia del lenguaje.

\section{Abstract}

The opening of spaces for the disabled person is tangible if it is generated from within formal and informal organizations. It requires a permanent work in the structuring of intergroup designs, institutional credibility and an intentionality of the communities that share the problematic of the individual and that combine efforts for a qualification of his life in a plane of equity with respect to his fellows. To this end, the present study is carried out in order to deal with the topic of language therapy. It deals with aspects related to language, its characteristics, and disorders. Definition, goals of language therapy, therapeutic skills and some research results.

Keywords: language; therapy; language therapy.

\section{Resumo}

Abrindo espaços para a pessoa com deficiência é tangível se ele é gestado a partir de dentro das organizações formais e informais. Ela exige um trabalho permanente na estruturação de projetos intergrupais, credibilidade institucional e intenções das comunidades que partilham os problemas do indivíduo e para juntar forças para uma qualificação de sua vida em um plano de equidade em relação aos seus pares. Para o efeito, o presente estudo, a fim de abordar a questão da terapia da fala é realizada nos mesmos aspectos relacionados com a língua, as características, os problemas são resolvidos. Definição, objetivos da terapia da fala, habilidades terapêuticas e alguns resultados da investigação.

Palavras chave: linguagem; terapia; terapia da fala. 


\section{Introducción}

El origen y la evolución del lenguaje sigue siendo un tema no resuelto en la actualidad por paleoantropólogos y lingüistas, que se ven obligados a basar sus estudios en evidencias indirectas debido a que el lenguaje es virtualmente invisible en el registro arqueológico. Las primeras hipótesis ya se iniciaron en los tiempos de Darwin.

En ese mismo sentido el lenguaje es uno de los rasgos de conducta más distintivos de nuestra especie, ya que ningún otro animal se comunica de la manera que lo hacemos nosotros. Por este motivo, sólo la comunicación humana puede ser llamada 'lenguaje', siendo los sistemas de comunicación animales 'medios de transmisión de información no lingüísticos'. Así pues, debemos descartar que el lenguaje sea solamente una forma superior de alguna función animal: el lenguaje es un fenómeno cualitativamente nuevo y, como tal, no tiene verdadero análogo dentro del mundo animal.

El ser humano tiene una tendencia instintiva a hablar, y en la actualidad, se sabe con certeza que el lenguaje evolucionó dentro del linaje humano, pero se sigue cuestionando cuando se originó y como tuvo lugar esa evolución. Existen diversas teorías acerca de la evolución histórica del lenguaje, la misma ha tenido distintos grados sucesivos, es decir, el camino que nos conduce a él es la suma de una serie muy diversa de aptitudes comunicativas cuya filogénesis se extiende, al menos, a dos millones de años atrás. (Campillo-Valero D 2005).

\section{Definición de lenguaje}

El concepto de lenguaje puede ser entendido como un recurso que hace posible la comunicación. En el caso de los seres humanos, esta herramienta se encuentra extremadamente desarrollada y es mucho más avanzada que en otras especies animales, ya que se trata de un proceso de raíces fisiológicas y psíquicas. El lenguaje, le brinda la posibilidad al hombre de seleccionar, citar, coordinar y combinar conceptos de diversa complejidad. (Pérez Porto J, Merino M 2012).

Significa entonces (Romero M. 2012) que el lenguaje es una capacidad o facultad extremadamente desarrollada en el ser humano; un sistema de comunicación más especializado que los de otras especies animales, a la vez fisiológico y psíquico, que pertenece tanto al dominio individual como al social y que nos capacita para abstraer, conceptualizar y comunicar. 


\section{Características del lenguaje y funciones.}

El lenguaje puede ser: racional, arbitrario, doblemente articulado, universal, adquirido, convencional y voluntario.

Con relación a sus funciones se destacan: representativa, informativa o referencial, emotiva o expresiva, apelativa o imperativa, fática, metalingüística. (El Lenguaje: Características y Funciones. 2017).

\section{Trastornos del lenguaje: Retraso del lenguaje.}

Como su nombre lo indica, existe un retardo en la adquisición de las diferentes etapas del desarrollo cronológico del lenguaje infantil. Debe considerarse a un niño con retraso en el lenguaje, cuando lo cuantitativo y lo cualitativo de su uso verbal se halle por debajo de la cifra media de los otros niños de su edad, es decir, cuando un niño continúa dependiendo de gestos para comunicarse cuando ya debiera estar utilizando signos convencionales verbales; cuando aun usando palabras lo hace de una manera tan deformada en su articulación que es ininteligible su lenguaje, y cuando sólo utiliza determinados elementos gramaticales tanto en el aspecto sintáctico como en el léxico, y en el aspecto pragmático, demorándose en la adquisición de otros nuevos elementos lingüísticos. (López Betancourt M. 2015).

Se clasifican según su etiología en:

- Retrasos primarios del desarrollo del lenguaje: El retraso primario del desarrollo del lenguaje está caracterizado principalmente por un retardo de la adquisición de las habilidades lingüísticas de acuerdo a la edad cronológica,

- Retrasos secundarios del desarrollo del lenguaje: El retraso secundario del desarrollo del lenguaje puede ser debido a diferentes causas pero las que primero hay que descartar, pues son las que tienen peor pronóstico, son las siguientes: Retraso mental, Lesiones del SNC o Periférico como la Parálisis cerebral entre otras. (López Betancourt M. 2015).

Son varias las razones por las cuales se debe valorar el lenguaje infantil, en primer lugar porque el habla es un indicador del desarrollo integral del niño, en segundo lugar, para detectar las dificultades en este proceso, y en tercer lugar, para determinar los niños en riesgo, así como para evaluar el progreso del niño sometido a tratamiento. (López Betancourt M. 2015). 
Clasificación de trastornos del habla: trastornos de articulación, trastornos con la fluidez del habla con problemas que incluyen tartamudez, resonancia o trastornos de la voz, disfagia oral/trastornos de la alimentación.

Los mismos pueden ser expresivos o receptivos.

Los trastornos del lenguaje y la comunicación suelen incidir, con frecuencia, de forma negativa, en múltiples dimensiones de la vida del individuo, desde su autoestima, relaciones sociales, estado de ánimo o su adaptación laboral.

En la actualidad existen multitud de estudios que corroboran un aumento de la prevalencia de dificultades asociadas al lenguaje, permanentes o transitorias, en niños diagnosticados con “necesidades educativas especiales" (Acosta, 2004).

\section{Terapia del lenguaje}

La Terapia de Lenguaje es el área de rehabilitación que trabaja con niños, que presentan dificultades del habla, lenguaje y/o comunicación, problemas con la producción de sonidos, o dificultades con el aprendizaje del lenguaje, es decir, fallas al combinar las palabras para expresar ideas.

El objetivo principal de la Terapia de Lenguaje, es establecer o restablecer la comunicación lingüística no desarrollada, alterada o interrumpida en el niño en donde con vocalizaciones, estrategias divertidas y juegos de acuerdo a su edad, lo estimularán para utilizar su lenguaje.

- Evaluar, diagnosticar y atender las deficiencias de lenguaje que presentan los niños.

- Promover el desarrollo integral de los niños con problemas de comunicación.

- Fomentar la participación de los padres para que sean colaboradores activos en programas terapéuticos de sus hijos.

- Ofrecer asesoría en todo lo relacionado con el desarrollo normal y la estimulación del lenguaje. (Terapia de Lenguaje ¿Qué es? 2014). 


\section{Habilidades terapéuticas en terapia de lenguaje.}

El terapeuta del lenguaje es la persona que dispone de un caudal de conocimientos relacionados con los problemas del habla y del lenguaje y que está entrenada para aplicar esos conocimientos a la solución de problemas clínicos. En sus funciones se distinguen diferentes actividades como la evaluación, el tratamiento, pero también el consejo a los pacientes y a su familia, así como orientar y proporcionar información a profesores y miembros de otras disciplinas relacionadas con los problemas de lenguaje y comunicación de su paciente. (Fernández Zúniga A, De León M. 2008).

La labor del logopeda, por tanto, implica, además del conocimiento específico sobre el diagnóstico y tratamiento de diferentes alteraciones, saber cómo manejar situaciones problemáticas de estrés o emocionales de sus pacientes, los cuales requieren de su orientación y apoyo. (Fernández Zúñiga A, De León M. 2008).

Desde esta perspectiva, el terapeuta del lenguaje no tiene sólo que centrarse en el paciente, en el niño o adulto, sino también en las personas que se encuentran alrededor.

En el aprendizaje del lenguaje, además de los aspectos específicos, se deben tener en cuenta el contexto en el que se enseña, la regularidad con que se practica y el estímulo que la persona recibe para comunicarse. Además, en el proceso de adquisición y desarrollo del lenguaje se consideran lógicamente también los interlocutores, los padres, la familia y el medio en el que el individuo se desenvuelve. (Fernández Zúñiga A, De León M. 2008).

Las habilidades terapéuticas permiten que el profesional adquiera las destrezas necesarias para establecer la relación interpersonal con el paciente, en la cual busque proporcionar a la persona o a la familia comprensión sobre el problema de lenguaje o la comunicación que padece y la forma de manejarlo, adaptarse a la situación y saber cómo hacer frente a ello. (Fernández Zúñiga A, De León M. 2008).

Dentro de las diferentes teorías o escuelas de consejo terapéutico, todas plantean que el terapeuta debe contar con una serie de características y actitudes, las cuales son similares en la mayoría de ellas, y se consideran importantes en el establecimiento de una relación terapéutica efectiva. (Fernández Zúñiga A, De León M. 2008). 
El Terapeuta del Lenguaje, Fonoaudiólogo o Logopeda (Pardo N. 2014), participa en la planeación y ejecución de acciones comunitarias de prevención, detección, diagnóstico e intervención en las áreas de lenguaje, habla y audición, con base en criterios científicos sólidos, propios o producto del desarrollo inter o transdisciplinario e instrumentación especializada, en los contextos educativo, industrial y de salud. Igualmente se interesa por realizar investigaciones que contribuyan al fortalecimiento cognoscitivo y ético de su profesión.

\section{Resultados de investigaciones acerca de la terapia del lenguaje.}

La situación de la investigación en relación con el tratamiento del Trastorno Específico del Lenguaje (TEL) Vera Giménez J (2010), expresa que existe una gran descompensación del esfuerzo investigador realizado cuando se compara con otros trastornos. Además de constatarse el hecho de una insuficiencia de estudios epidemiológicos del trastorno, se corrobora la escasez de trabajos rigurosos sobre su tratamiento que han permitido ser en orden a organizarlos con arreglo a diferentes criterios que facilitan la conclusión de los modelos de intervención, centrados en el tratamiento de componentes específicos del lenguaje más que los modelos generalistas se revelan como más eficaces en la remisión de las consecuencias del TEL, especialmente si se llevan a cabo en contextos de intervención directa -aplicada por el especialista- y contextual -realización en entorno conversacional, facilitador de la incorporación por imitación voluntaria.

Law J (2007), en su estudio el cual tuvo como finalidad analizar la efectividad de las intervenciones del habla, y el lenguaje en niños con retraso o trastorno primario del habla y el lenguaje. Indica que hay un efecto positivo en las intervenciones de la terapia del habla y el lenguaje en niños con dificultades en la expresión fonológica y del vocabulario. Las pruebas para las dificultades en la expresión de sintaxis son más contradictorias, y se necesitan más investigaciones para estudiar la intervención para las dificultades en la recepción del lenguaje.

Según lo expuesto en una investigación realizada por Ato Lozano (2009) y colaboradores. Los resultados muestran que la intervención centrada en la familia produce mejoras en el rendimiento lingüístico cuanto menos similares a los de la terapia habitual administrada por un profesional. Además, los beneficios de esta intervención no son sólo relativos al área del lenguaje, sino también al área psicológica y emocional de todos los miembros de la familia. 
La tesis realizada por Vargas Cochancela y Zeas Tenemaza (2013), acerca de la evaluación de una intervención logopédica del retraso simple en el desarrollo de lenguaje en niños y niñas de 2 a 4 años de edad, Cuenca-Ecuador, concluyó que la prevalencia general del trastorno simple en el desarrollo del lenguaje fue de $26.1 \%$, el tratamiento dado mejoró en un $100 \%$.

La dislalia es uno de los trastornos del habla más frecuentes que se presentan en los niños de edad escolar. En el Ecuador, según los resultados de una investigación que culminó en 1996, auspiciada por el CONADIS, INNFA y el Instituto de Migración y Servicios Sociales, (IMSERSO de España) el 6\% de discapacidades en niños mayores de cinco años corresponden a los trastornos del lenguaje. Los resultados de la investigación ejecutada por Peñafiel Sari K. (2010), posterior a la intervención Logopédica, el 13,7\% de las niñas y niños re-evaluados superaron la dificultad articulatoria y el $12,4 \%$ no la superaron, persistiendo principalmente en los fonemas /d/, /s/, /r/, /rr/, en diptongos, en dífonos y en sílabas inversas, esto se debe a que son fonemas que presentan una compleja articulación, lo que hace que su adquisición sea más tardía con respecto a los demás fonemas. La intervención logopédica es el pilar fundamental posterior a la detección de una Dislalia Funcional para una buena rehabilitación de los trastornos del habla.

La Estimulación Temprana en el desarrollo del lenguaje oral es un tratamiento con bases científicas durante los primeros años de vida, encaminado a potencializar al máximo las posibilidades físicas e intelectuales de el/la niño/a, para que pueda desenvolverse mejor en su vida y resolver sus necesidades e inquietudes por lo que tiene una gran importancia. Un estudio realizado a los niños y niñas del Primer Año de Educación General Básica de la Escuela Heredia Bustamante de la ciudad de Quito. Los resultados obtenidos determinaron las necesidades específicas que tienen los niños/as para mejorar la fluidez verbal a nivel fonológico, sintáctico y semántico. (Verdezoto Campaña M C. 2011).

Por último la intervención logopédica es de gran importancia para el establecimiento de la comunicación y el lenguaje oral, escrito y/o facilitando en las diferentes situaciones de la vida y su contribución al desarrollo de la personalidad; suscitar necesidades de comunicación oral y escrita en diferentes contextos y estimular los procesos psicológicos que están en la base de los mecanismos expresivos, así como los procesos de comprensión y producción de significados. 


\section{Referencias bibliográficas}

Acosta, V.M. (2004). Las prácticas educativas ante las dificultades del lenguaje. Una propuesta desde la acción. Barcelona: Ars Médica.

Ato Lozano, E; Galián Conesa, M M D; Cabello Luque, F. (2009).Intervención familiar en niños con trastornos del lenguaje: Una revisión. Electronic Journal of Research in Educational Psychology. 7. (19), pp. 1419-1448. [Consulta 12 agosto 2016]. Recuperado de: http://www.redalyc.org/articulo.oa?id=293121984021

Campillo-Valero D, Garcia-Guixé E. (2005). Origen y evolución del lenguaje. REV NEURO; 41 (Supl 1): S5-S10.

El Lenguaje: Características y Funciones. (2017). [Consulta 12 agosto 2016]. Recuperado de https://10conceptos.com/el-lenguaje-caracteristicas-y-funciones/

Fernández Zúñiga A, De León M. (2008). Habilidades terapéuticas en terapia de lenguaje. Relación terapeuta-paciente. Revista de Logopedia, Foniatría y Audiología 28( 1), 34-45

López Betancourt M. (2015). Retraso del lenguaje. [Consulta 12 agosto 2016]. Recuperado de www.sld.cu/galerias/pdf/sitios/prevemi/retraso_del_lenguaje_1.pdf

Law J, Garrett Z, Nye C (2007). Intervenciones de terapia del habla y el lenguaje para niños con retraso o trastorno primario del habla y el lenguaje. Recuperado de http://www.updatesoftware.com

Pérez Porto J, Merino M (2012). Definición de lenguaje. [Consulta 26 agosto 2016]. Recuperado de http://definicion.de/lenguaje/

Pardo N. (2014). La terapia del lenguaje, fonoaudiología o logopedia y la transdisciplinaridad. [Consulta 26 agosto 2016]. Recuperado de http://fonoaudiologia.com/artigo/la-terapia-dellenguaje-fonoaudiologia-o-logopedia-y-la-transdisciplinaridad.html

Peñafiel Sari K (2010). Evaluación e intervención logopédica de dislalias funcionales en niños y niñas de primero a tercer año de educación básica, Escuela Fiscal Mixta "Panamá", noviembre 2009 - mayo 2010". Cuenca - Ecuador. Recuperado de dspace.ucuenca.edu.ec/bitstream/123456789/3804/1/TECF59.pdf 
Romero M. (2012). Lenguaje y comunicación. [Consulta 26 agosto 2016]. Recuperado de http://lenguajeycomunicacionunefa.blogspot.com/2012/11/el-lenguaje-definicion.html

Terapia de Lenguaje ¿Qué es? (2014). [Consulta 26 agosto 2016]. Recuperado de http://reconstruye.com/2014/09/12/terapia-de-lenguaje-que-es/

Vera Giménez J. (2010). El tratamiento del trastorno específico del lenguaje: estudio de $\begin{array}{lllll}\text { situación. } \quad \text { [Consulta } & 04 & \text { julio 2016]. Recuperado de }\end{array}$ https://uvadoc.uva.es/bitstream/10324/1853/1/ponencia_jesus_vera.pdf

Vargas Cochancela L, Zeas Tenemaza J (2013). Evaluación de una intervención logopedica del retraso simple en el desarrollo de lenguaje en niños y niñas de 2 a 4 años de edad. Cuenca Ecuador. [Consulta 04 julio 2016]. Recuperado de dspace.ucuenca.edu.ec/bitstream/123456789/4955/1/TECF26.pdf

Verdezoto Campaña M C. (2011). La estimulación temprana en el desarrollo del lenguaje oral, de los niños y niñas del primer año de educación básica, de la escuela Heredia Bustamante de la Ciudad de Quito durante el año lectivo 2010-2011. [Consulta 04 julio 2016]. Recuperado de www.dspace.uce.edu.ec/bitstream/25000/272/1/T-UCE-0010-52.pdf 